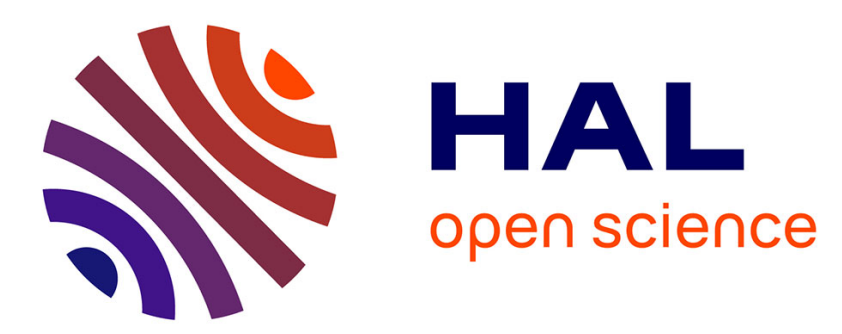

\title{
Multi-scale acoustics of partially open cell poroelastic foams
}

\author{
Minh Tan Hoang, Guy Bonnet, Camille Perrot
}

\section{To cite this version:}

Minh Tan Hoang, Guy Bonnet, Camille Perrot. Multi-scale acoustics of partially open cell poroelastic foams. The 21st International Congress on Acoustics (ICA 2013), Jun 2013, Montréal, Canada. pp.065013, 10.1121/1.4799694 . hal-00832543

\section{HAL Id: hal-00832543 \\ https://hal.science/hal-00832543}

Submitted on 9 Jun 2015

HAL is a multi-disciplinary open access archive for the deposit and dissemination of scientific research documents, whether they are published or not. The documents may come from teaching and research institutions in France or abroad, or from public or private research centers.
L'archive ouverte pluridisciplinaire HAL, est destinée au dépôt et à la diffusion de documents scientifiques de niveau recherche, publiés ou non, émanant des établissements d'enseignement et de recherche français ou étrangers, des laboratoires publics ou privés. 


\section{Proceedings of Meetings on Acoustics}
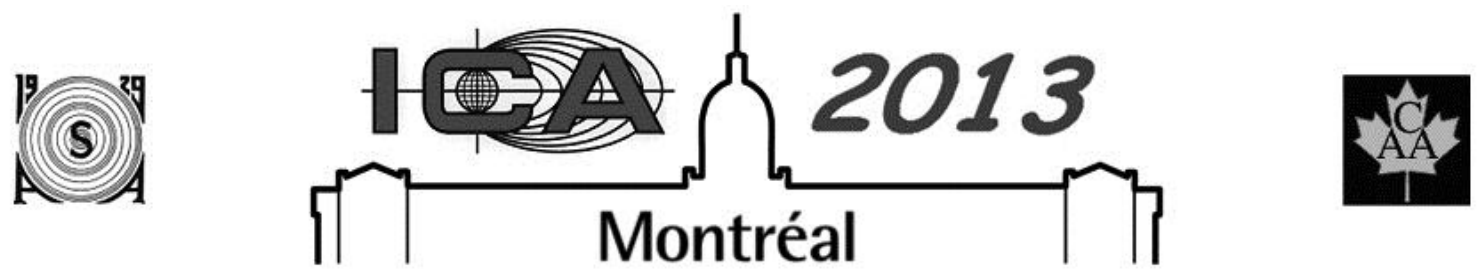

ICA 2013 Montreal

Montreal, Canada

2 - 7 June 2013

Structural Acoustics and Vibration

Session 1pSA: Measurement and Modeling of Structures with Attached Noise Control Materials II

\section{1pSA4. Multi-scale acoustics of partially open cell poroelastic foams}

Minh Tan Hoang, Guy Bonnet and Camille Perrot*

*Corresponding author's address: Laboratoire Modélisation et Simulation Multi Echelle, Université Paris-Est, MSME UMR 8208

CNRS, Marne-la-Vallée, 77454, --, France, camille.perrot@univ-paris-est.fr

The present paper reports on the modeling of linear elastic properties of acoustically insulating foams with unit cells containing solid films or membranes at the junction between interconnected pores from a numerical homogenization technique. It combines fluid-flow induced microstructure identification with simulations of the effective Young's modulus and Poisson ratio from a mixture of routinely available laboratory measurements (porosity, permeability, cell size) and finite element calculations when the boundary conditions of the periodic unit cell take particular symmetric forms. This combination results in microstructural determination of the macroscopic coefficients entering into the Biot-Allard theory of wave propagation and dissipation through porous media. Precise control over pore morphology and mechanical properties of the base material renders this multi-scale approach particularly suitable for various advanced applications.

Published by the Acoustical Society of America through the American Institute of Physics 


\section{INTRODUCTION}

Foams and cellular materials are very useful in the fields of building and transportation industries for sound proofing applications. ${ }^{1}$ Those containing both an interconnected fluid phase and a deformable solid phase are highly desirable from the standpoint of pore accessibility (visco-thermal dissipations) and structural isolation (decoupling effect). Although semi-phenomenological approaches of modeling this type of materials have dominated the landscape, ${ }^{2-4}$ multi-scale methods ${ }^{5-9}$ have received attention due to the need to improve their acoustical properties using a synthetic control of their microstructure. Partially open-cell solid foams are an interesting class of cellular solids that are versatile precursors to advanced poroelastic materials with well-defined pore structures, sizes, and interface functionality. ${ }^{10-11}$ However, there are three key issues that currently limit the utility of partially open-cell foams in sound proofing application. The straightforward calculation of elastic properties with a well-defined pore structure has only been achieved in a few cases. ${ }^{12-15}$ Modeling the closure rate of membranes at the junction between interconnected pores has been challenging due to the non-homogeneity of the membrane closure rate itself.

${ }^{16-17}$ In addition, fluid and solid mechanics at the cell size level that determine macroscopic transport and mechanical responses of foam are generally considered separately. Here, we present a new solid foams membrane-based strategy for the performance prediction of advanced poroelastic materials that simultaneously address all three of these issues. Our main objective is to perform numerical homogenization simulations of linear elastic properties by the finite element technique in solid foams containing membranes with prescribed closure rates, in order to derive Young's modulus and Poisson ratio for real foam samples with various reticulation rates. Assuming scale separation, such a numerical estimate enables us to derive elastic coefficients for describing acoustic wave propagation in poroelastic materials on a microstructural basis. ${ }^{8}$ At first, we used fluid-flow induced cellular morphology. Secondly, linear elastic properties were simulated by applying a version of periodic homogenization method ${ }^{18}$ from the standpoint of the polyhedral unit cells previously determined having cubic symmetry (namely non-elongated Kelvin cell models). Importantly, this second step is decoupled from the first one and corresponds to a quasi-static situation where the fluid is ignored. In a third step, acoustical properties of the poroelastic materials under study are simulated using either the analytical formulas derived by Biot, or a Biot axisymmetric finite-element formulation when the boundary conditions at the peripheral of the real porous samples have a significant influence on the overall result.

\section{MATERIALS AND METHODS}

\subsection{Regular Solid Foam Structure with a Specified Closure Rate of Membranes}

The spatial structure of the solid foam models proposed here is derived from routinely available laboratory measurements (permeability, porosity, ligament length) and transport processes simulation inside porous media. A general view on the representation of solid foams and on the generation processes can be found in the studies of Perrot et al. ${ }^{19}$ and Hoang and Perrot ${ }^{20-21}$. The solid foam models which are going to be used are spatially periodic in all directions of space. Hence, the unit cell provides the basic structure from which the whole medium can be derived by translation along three coordinate axes. Two different samples of solid foams were studied; let us consider them more precisely in Fig. 1 which summarizes their more important geometrical characteristics. The ordered networks, analyzed in this article and shown in Fig. 1, are truncated octahedron networks with tetrahedral vertices; the ligaments of circular cross-section shape connect the spherical center of a regular tetrahedron, with plates partially opened or not forming the faces of this polyhedron. This regular foam structure of cubic symmetry follows this geometry from an initially open-cell structure, whose close rate of solid films or membranes is allowed to grow. This implies that the length of the ligaments of the model becomes compatible with measurements on scanning electron micrographs (see Ref. 20 for further details).

\subsection{Purely Geometrical Macroscopic Properties}

The two samples, namely $\mathrm{H}_{1}$ and $\mathrm{H}_{2}$ were generated with the following measured porosity ${ }^{22}, \phi=0.93 \pm 0.01$ and $\phi=0.97 \pm 0.01$ respectively. The characteristic ligament length (thickness $2 r$ ) $L$ of the first sample $\mathrm{H}_{1}$ is smaller than the characteristic length (thickness) of the second sample $\mathrm{H}_{2}$ (See Fig. 1. of this paper and Eq. (1) of Ref. 19). 

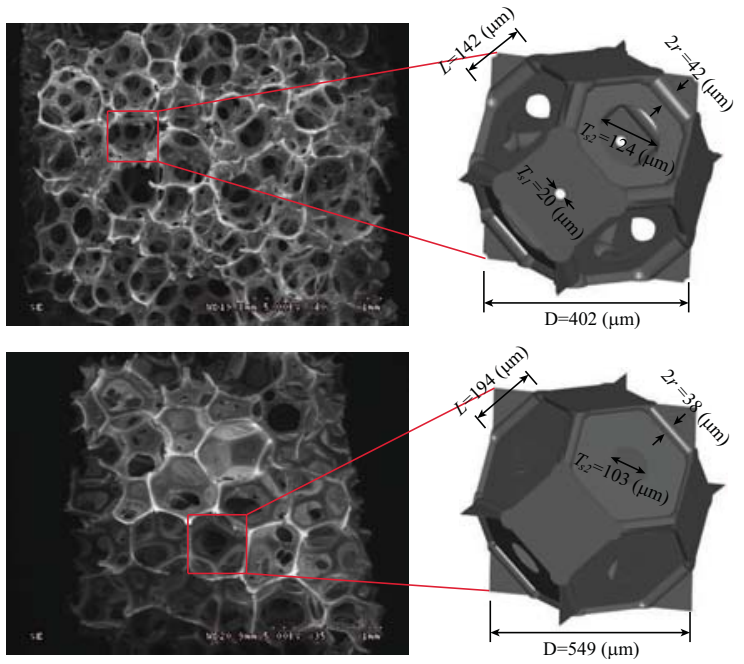

FIGURE 1. Geometrical characteristics of two different solid foam samples, $\mathrm{H}_{1}$ (top) and $\mathrm{H}_{2}$ (bottom).

The pore solid surface $S$ and pore volume $V_{p}$ were systematically calculated for each solid foam sample with elementary spatial integration. These two quantities can be combined in order to define the length scale $\Lambda^{\prime}=2 V_{p} / S$, a generalized hydraulic radius also named thermal characteristic length in the context of sound absorbing material. ${ }^{4}$

\subsection{Transport Parameters}

The macroscopic permeability $k_{0}$ of each solid foam sample was measured ${ }^{23}$ and computed on a unit cell by solving the Stokes equations by a finite element method. ${ }^{19-20}$ The size of the cell is adapted in order to recover the measured permeability. The viscous characteristic length $\Lambda$ can be used to characterize the throat size $T_{S}$ of a porous media. ${ }^{21} \Lambda$ was introduced by Johnson et $a l .{ }^{3}$ It is essentially a volume-to-surface pore ratio with a measure weighted by the local value of the electric field $\mathbf{E}(\mathrm{X})$ in a conduction process,

$$
\Lambda=\frac{2 \int_{V p}|\mathbf{E}(\mathrm{X})|^{2} d V}{\int_{S}|\mathbf{E}(\mathrm{X})|^{2} d S} .
$$

The tortuosity $\alpha_{\infty}$ can be calculated when the electric field is known,

$$
\alpha_{\infty}=\frac{\left\langle\mathbf{E}(\mathrm{X})^{2}\right\rangle}{\langle\mathbf{E}(\mathrm{X})\rangle^{2}},
$$

where $\langle\bullet\rangle$ denotes a fluid phase average. This quantity was also obtained from our computation on the unit cell.

\subsection{Effective Mechanical Properties}

The effective mechanical properties were obtained for the two different solid foam models either from both finite element calculations or from experiments on real samples, namely $\mathrm{H}_{1}$ and $\mathrm{H}_{2}$. The effective linear-elastic properties 

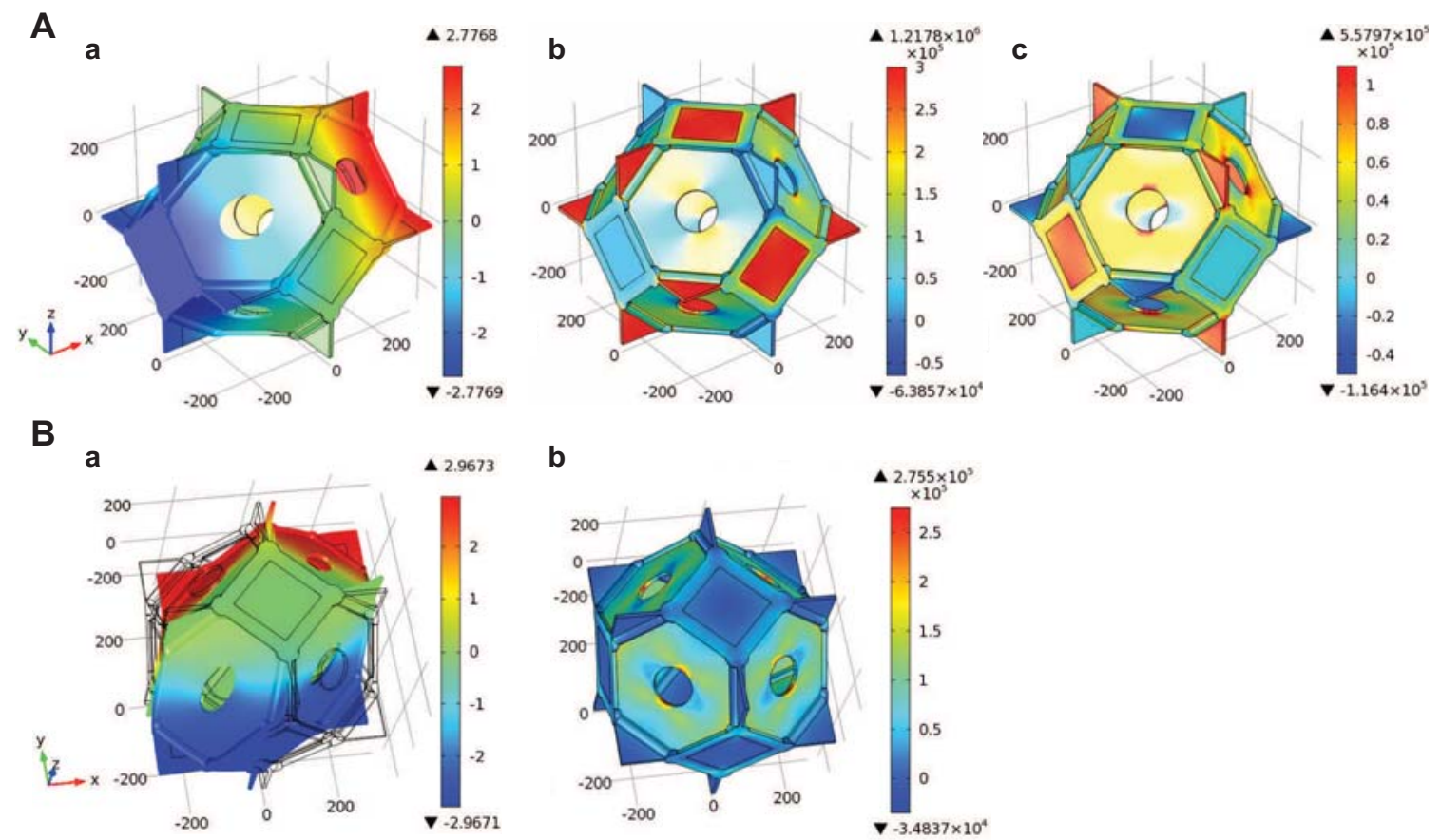

FIGURE 2. Numerical experiments allowing to identify the elastic constants $C_{11}, C_{22}$ and $C_{44}$ (in contracted notations) of the elasticity matrix for a solid foam model having cubic symmetry; illustrated with foam sample $\mathrm{H}_{2}$. (A) Tensile strain numerical experiment. A uniform strain vector $E=E_{11} \mathbf{e}_{1} \otimes \mathbf{e}_{1}$ is applied (along the principal coordinate direction [100]) in the equivalent homogeneous material with $E_{11}=0.01$ showing $(\mathrm{a})$ the displacement field $(\mu \mathrm{m})$, (b) the stress field $\sigma_{11}$ $\left(\mathrm{N} / \mathrm{m}^{2}\right)$ and $(\mathrm{c})$ the stress field $\sigma_{22}\left(\mathrm{~N} / \mathrm{m}^{2}\right)$. The data are then averaged over the periodic unit cell as follows: $\Sigma_{11}=<\sigma_{11}$ $>, \Sigma_{22}=\left\langle\sigma_{22}>\right.$, and $\Sigma_{33}=<\sigma_{33}>$. Elastic constants $C_{11}$ and $C_{12}$ are computed using the relations $\Sigma_{11}=C_{11} E_{11}$ and $\Sigma_{22}=$ $C_{12} E_{11}$ (or $\Sigma_{33}=C_{12} E_{11}$ ). With a transversely isotropic configuration, the effective Young's modulus was found to be unchanged; $E_{T I}=E_{L}$ and $v_{T I}=v_{L}$. Contrary to the transversely isotropic configuration, the effective Young's modulus and Poisson ratio for an isotropic material configuration were modified; $E_{I}$ differs from $E_{L}$. and $v_{T I}$ from $v_{L}$. (B) Shear strain numerical experiment. A uniform strain vector $E=E_{12}\left(\mathbf{e}_{1} \otimes \mathbf{e}_{2}+\mathbf{e}_{2} \otimes \mathbf{e}_{1}\right)$ is applied in the equivalent homogeneous material with $E_{12}=0.01$, showing (a) the displacement field $(\mu \mathrm{m})$ and $(\mathrm{b})$ the stress field $\sigma_{12}\left(\mathrm{~N} / \mathrm{m}^{2}\right)$. The data are then averaged over the periodic unit cell as follows: $\Sigma_{12}=\left\langle\sigma_{12}\right\rangle$. The last elastic constant $C_{44}$ is computed using the relation $\Sigma_{12}=C_{44} E_{12}$.

of the solid networks are determined by a finite element scheme operating on discretized representations of the structure. The material is assumed to be locally isotropic and linear-elastic; the fluid phase is modeled as a vacuum.

The effective elastic longitudinal modulus $E_{L}$ and Poisson ratio $v_{L}$ are obtained by applying two macroscopic external strains on the cube that bounds the solid foam model: a tensile strain and a shear strain related to the principal coordinate directions. Since the solid foam models have cubic symmetry, three independent elastic constants $C_{11}, C_{22}$ and $C_{44}$ (in contracted notations) exist in the elasticity matrix, whose identification requires two numerical experiments using periodic boundary conditions, to express $E_{L}$ and $v_{L}$. The effective elastic longitudinal modulus $E_{L}$ and Poisson ratio $v_{L}$ are obtained by applying the relations $E_{L}=\left(C_{11}^{2}+C_{11} C_{12}-2 C_{12}^{2}\right) /\left(C_{11}+C_{12}\right)$ and $v_{L}=C_{12} /\left(C_{11}+C_{12}\right)$ which are valid for the effective elastic properties along the principal coordinate directions, [100], [010] or [001] of materials with cubic symmetry.

The effective elastic properties (lower bounds) of an effective transversely isotropic material and of an effective isotropic material correspond to an average of the properties obtained by random orientation of the unit cell obtained by arbitrary rotation around a given axis or for any arbitrary rotation in space. In particular, the detailed expression obtained by this angular averaging procedure yields for the isotropic case : $E_{I}=\left[5\left(C_{11}-C_{12}\right)\left(C_{11}+2 C_{12}\right) C_{44}\right] /\left[C_{44}\right.$ $\left.\left(3 C_{11}+C_{12}\right)+2\left(C_{11}-C_{12}\right)\left(C_{11}+2 C_{12}\right)\right]$, and $v_{I}=\left[-\left(C_{11}-3 C_{12}\right) C_{44}+\left(C_{11}-C_{12}\right)\left(C_{11}+2 C_{12}\right)\right] /\left[C_{44}\left(3 C_{11}+C_{12}\right)+\right.$ $\left.2\left(C_{11}-C_{12}\right)\left(C_{11}+2 C_{12}\right)\right]$.

Fig. 2 shows the displacement and stress fields of the solid foam model corresponding to the real sample $\mathrm{H}_{1}$ during the shear strain and the tensile strain numerical experiments. 


\section{RESULTS AND DISCUSSION}

\subsection{Geometrical and Transport Properties}

Before looking at the mechanical results, it is of interest to consider the purely geometrical macroscopic properties $\left(\phi, \Lambda^{\prime}\right)$ and transport parameters $\left(k_{0}, \Lambda, \alpha_{\infty}\right)$ which were obtained on the cubic cell.

The experimental data $\left.\left(\phi, k_{0}, \Lambda, \Lambda^{\prime} \alpha_{\infty}\right)\right)$ were obtained at the Groupe d'Acoustique de l'Université de Sherbrooke (GAUS) laboratory (Québec, Canada). Three of these parameters were characterized using direct experimental techniques; $\phi^{22}, k_{0}{ }^{23}$ and $\alpha_{\infty}{ }^{25}$. Then, the determination of the missing parameters, $\Lambda^{\prime}$ and $\Lambda$, is based on an inverse procedure using a cost function minimization algorithm. The principle of the method consists in minimizing the differences between measured and modeled sound absorption curves, from a standing wave tube setup and Johnson-Champoux-Allard ${ }^{3-4}$ model respectively (FoamX software ${ }^{26}$ ).

The purely geometrical macroscopic properties $\left(\Lambda^{\prime}\right)$ and transport parameters $\left(\Lambda, \alpha_{\infty}\right)$ computed from the course of this multi-scale approach are in a good agreement with independently obtained experimental data, especially when standard deviations are taken into account as seen in Table 1 . This means that the identified microstructures presented in Fig. 1 are representative of the macroscopic properties of the real porous materials under study from both purely geometrical macroscopic quantities and transport processes point of views within experimental uncertainties. We shall now try to examine the representativity of these microstructures in terms of their effective linear poroelastic properties.

TABLE 1. Macroscopic geometrical and transport parameters, experiments vs numerical results.

\begin{tabular}{|c|c|c|c|c|c|c|}
\hline Foam & Method & $\phi(-)$ & $\Lambda^{\prime}(\mu \mathrm{m})$ & $k_{0}\left(\times 10^{-10} \mathrm{~m}^{2}\right)$ & $\Lambda(\mu \mathrm{m})$ & $\alpha_{\infty}(-)$ \\
\hline \multirow{4}{*}{$\mathrm{H}_{1}$} & Computation & & $139 \pm 26$ & & $44 \pm 5$ & $1.58 \pm 0.33$ \\
\hline & Direct characterization & $0.93 \pm 0.01$ & & $3.87 \pm 0.32$ & & $2.44 \pm 0.57$ \\
\hline & Inverse characterization & $0.93 \pm 0.01$ & $136 \pm 36$ & $3.87 \pm 0.32$ & $45 \pm 16$ & $2.44 \pm 0.57$ \\
\hline & Measurements & & & & & \\
\hline \multirow{3}{*}{$\mathrm{H}_{2}$} & Computation & & $171 \pm 48$ & & $41 \pm 6$ & $2.79 \pm 0.61$ \\
\hline & Direct characterization & $0.97 \pm 0.01$ & & $1.59 \pm 0.31$ & & $1.24 \pm 0.05$ \\
\hline & $\begin{array}{l}\text { Inverse characterization } \\
\text { Measurements }\end{array}$ & $0.96 \pm 0.01$ & $103 \pm 108$ & $1.85 \pm 0.71$ & $48 \pm 19$ & $4.45 \pm 1.48$ \\
\hline
\end{tabular}

\subsection{Linear Elastic Properties}

The basic ingredients of flexible urethane foams of the type considered in this study are ester resin (or polyol), diisocyanate, water, catalysts and surfactants. ${ }^{29}$ The samples represent cylindrical subsections of large panels (of diameter equal to $44.5 \mathrm{~mm}$; and height equal to $10 \mathrm{~mm}, 15 \mathrm{~mm}$, and $20 \mathrm{~mm}$ for $\mathrm{H}_{2} ; 25 \mathrm{~mm}$ for $\mathrm{H}_{1}$ ).

Accurate literature values for the microscopic Young's modulus $E_{\mu}$ and Poisson ratio $v_{\mu}$ are not available because these values are depending on processing strategy. The values obtained in the literature are scattered within a range of more than one order of magnitude, lying between 2 and $30 \mathrm{MPa}^{30}$. As mentioned by Gong et al. ${ }^{12}$, some foam chemists believe that the polymer flow resulting from the foaming process may cause preferential alignment of the long molecules of the material along the ligaments. Since these characteristics may not be easily achievable in bulk material, they recommend that the mechanical properties of the polymer be measured directly from foam ligaments. To do so, a modified microscopic testing stage was used by Gong et al., to test ligaments of a 3 ppi foam sample $\left(E_{L}\right.$ $=4.5 \pm 0.3 \mathrm{MPa}$ ). However, the main conclusion which can be drawn is that specific measurements must be made on the material. Without these measurements at the present time, the range 2-30 MPa will be kept in the following.

Due to the strong dependence of the foam properties, all results of the computations performed along the lines of the previous section were given by values of relative Young's moduli $E / E_{p u}$, where $E_{\mu}=E_{p u}$ is the Young's modulus of the polyurethane, in Table 2 (superscript ${ }^{\text {a }}$, corresponds to non-dimensional values).

These values were first computed with membranes. However, a parameter which has a strong effect on the results is the membrane thickness. This one was obtained by optical microscopy, but the thickness is probably not constant and the observation of thickness can also be overestimated through microscopic observation. So, two results are given in Table 2: the one with the geometry of membranes as given by observation, and the one without membrane. 
TABLE 2. Computed relative elastic properties $E / E_{p u}$ of foam samples $\mathrm{H}_{1}$ and $\mathrm{H}_{2}$.

\begin{tabular}{llcccccccccc}
\hline \hline Foam & Method & $\begin{array}{c}\Sigma_{11}^{a} \\
\left(\times 10^{-3}\right)\end{array}$ & $\begin{array}{c}\Sigma_{22}^{a} \\
\left(\times 10^{-3}\right)\end{array}$ & $\begin{array}{c}\Sigma_{12}^{a} \\
\left(\times 10^{-3}\right)\end{array}$ & $\begin{array}{c}C_{11}^{a} \\
\left(\times 10^{-3}\right)\end{array}$ & $\begin{array}{c}C_{12}^{a} \\
\left(\times 10^{-3}\right)\end{array}$ & $\begin{array}{c}C_{44}^{a} \\
\left(\times 10^{-3}\right)\end{array}$ & $\begin{array}{c}E_{L}^{a} \\
\left(\times 10^{-3}\right)\end{array}$ & $\begin{array}{c}v_{L} \\
(-)\end{array}$ & $\begin{array}{c}E_{I}^{a} \\
\left(\times 10^{-3}\right)\end{array}$ & $\begin{array}{c}v_{\mathrm{I}} \\
(-)\end{array}$ \\
\hline \hline & Without & 0.12 & 0.09 & 0.03 & 12.44 & 9.04 & 2.91 & 4.83 & 0.42 & 4.4 & 0.43 \\
$\mathrm{H}_{1}$ & membranes & \pm 0.02 & \pm 0.01 & \pm 0.01 & \pm 2.30 & \pm 1.25 & \pm 0.86 & \pm 1.44 & \pm 0.01 & \pm 1.29 & \pm 0.01 \\
& With & 0.40 & 0.15 & 0.17 & 39.51 & 15.09 & 17.12 & 31.17 & 0.28 & 25.60 & 0.32 \\
& membranes & \pm 0.03 & \pm 0.01 & \pm 0.02 & \pm 2.63 & \pm 1.04 & \pm 1.90 & \pm 2.14 & \pm 0.01 & \pm 2.19 & \pm 0.01 \\
\hline \multirow{4}{*}{$\mathrm{H}_{2}$} & Without & 0.044 & 0.038 & 0.005 & 4.39 & 3.84 & 0.50 & 0.81 & 0.47 & 0.76 & 0.47 \\
& membranes & \pm 0.019 & \pm 0.015 & \pm 0.004 & \pm 1.89 & \pm 1.46 & \pm 0.38 & \pm 0.63 & \pm 0.01 & \pm 0.59 & \pm 0.01 \\
& With & 0.30 & 0.13 & 0.17 & 30.21 & 12.64 & 16.74 & 22.75 & 0.30 & 22.18 & 0.30 \\
& membranes & \pm 0.07 & \pm 0.02 & \pm 0.03 & \pm 6.81 & \pm 2.21 & \pm 3.00 & \pm 5.81 & \pm 0.01 & \pm 4.61 & \pm 0.00 \\
\hline \hline
\end{tabular}

The experimental values of Young's modulus are given in Table 3. They are obtained by uniaxial compression experiments ${ }^{27-28}$ on polyurethane foam samples, namely $\mathrm{H}_{1}$ and $\mathrm{H}_{2}$; see Fig. 1. For comparison with the computed results, the relative Young's moduli of both foams are given for both ultimate values of $E_{p u}(2-30 \mathrm{MPa})$. These values can be compared on the same table with relative Young's moduli coming from the computations.

Obviously, the range of experimental relative moduli follows the one of polyurethane modulus. However, some conclusions can still be drawn. It can be seen for $\mathrm{H}_{1}$ that the estimation of the relative modulus with membranes does not lie between the values given by experiment, as the relative modulus without membranes is compatible with these values. It means that the membrane thickness used for this foam is probably overestimated when constructing the periodic cell. On the contrary, the value of relative Young's modulus obtained for $\mathrm{H}_{2}$ without membranes is clearly outside the admissible range of relative moduli. It means that the membranes must be taken into account in estimating the Young's modulus of the foam. This last result confirms the need to take into account the membranes when estimating the elastic properties of such foams.

TABLE 3. Computed relative elastic properties $E / E_{p u}$ of foam samples $\mathrm{H}_{1}$ and $\mathrm{H}_{2}$.

\begin{tabular}{cccccc}
\hline \hline Foam & $\begin{array}{c}1000 E_{\text {exp }} \\
(\mathrm{MPa})\end{array}$ & $1000 E_{\text {exp }} / E_{p u(\min )}$ & $1000 E_{\text {exp }} / E_{p u(\max )}$ & $\begin{array}{c}1000 E_{\text {comp }} / E_{p u} \\
\text { (with membranes) }\end{array}$ & $\begin{array}{c}1000 E_{\text {comp }} / E_{p u} \\
\text { (without membranes) }\end{array}$ \\
\hline $\mathrm{H}_{1}$ & 18 & 9 & 0.6 & 25.6 & 4.4 \\
$\mathrm{H}_{2}$ & 111 & 55 & 3.7 & 22.2 & 0.76 \\
\hline \hline
\end{tabular}

\subsection{Acoustical properties}

Let us go further to one of the major purpose of this study, namely the determination of the acoustical properties of the poroelastic medium. The multi-scale determination of purely geometrical parameters, transport, and elastic properties was studied for the regular packing of partially open cell structures for two samples of solid foams. The numerical and experimental data relative to these macroscopic parameters are gathered in Tables 1 and 3 . These results can be used as input parameters of approximate but robust semi-phenomenological models ${ }^{3-4}$ ("universal curves") as summarized in Appendix B of Ref. 19. We shall focus the presentation on the sound absorption $\alpha(\omega)$ and sound transmission STL $(\omega)$ properties relative to the reconstructed poroelastic media (and we shall point out the features specific to each of these solid foams, $\mathrm{H}_{1}$ and $\mathrm{H}_{2}$ ).

Some results relative to $\alpha(\omega)$ and STL $(\omega)$ are displayed in Fig. 3. It is seen from this figure that most of the sound absorbing behavior of these foams is captured from the multi-scale approach, with practically no need to introduce the elastic properties of the real foam samples. In Fig. 3A(a), it is seen that the acoustical behavior of foam sample $\mathrm{H}_{1}$ (of density $\rho_{\mathrm{H} 1}=68 \mathrm{~kg} / \mathrm{m}^{3}$, whereas $\rho_{\mathrm{H} 2}=25 \mathrm{~kg} / \mathrm{m}^{3}$ ) is well described by a limp model ${ }^{32}$ [foam sample of Fig 1(top)]. It is possible to verify this property with the sound transmission loss measured from a standing wave tube, since $\lim _{\omega \rightarrow 0} S T L(\omega) \simeq 0$, see Fig. $3 \mathrm{~A}(\mathrm{~b})$. In other words, the rigid vibrational mode of the foam sample $\mathrm{H}_{1}$ is excited at $0 \mathrm{~Hz}$, and $S T L(\omega=0)=0$. Therefore, the elastic properties of the porous material are practically useless. 
For sample $\mathrm{H}_{2}$, because of the low permeability of the membrane-based porous material [the foam sample of Fig. 1(down)], the corresponding Frame Acoustical Excitability (FAE) ${ }^{31}$ is high, and the skeleton is significantly excited by the acoustic wave. Accordingly, a loss in the sound absorption spectrum is observed around the skeleton resonances, and especially around the first one; Fig. 3B(a). Furthermore, the sound transmission loss behavior around $0 \mathrm{~Hz}$ depends on the boundary conditions type at the peripheral of the material (sliding or bounded) according to the compressional rate exerted by the tube on the porous sample. In the bounded case, the STL starts at a value differing from zero because the stiffness effect is significant. Here, one can see on Fig. 3B(b) that the STL starts at $10 \mathrm{~dB}$ at zero Hertz, and that a drastic loss is observed corresponding to the first resonance frequency. A 2D elastic model is therefore the more adapted for this configuration. Alternatively, if one wants to use an equivalentfluid model, a rigid model is required in the low frequency range (frequency domain governed by the stiffness) and a limp one after the first resonance (frequency domain governed by the mass). It is seen that the elastic properties affect the frequency-dependent functions $\alpha(\omega)$ and STL $(\omega)$.

These results show indirectly the usefulness of including the membranes in the estimation of Young's modulus. Indeed, without membranes, the estimation of Young's modulus would lead to reject the assumption of rigid skeleton, while this one is perfectly predicted by taking into account the membranes in the computation of the foam's Young's modulus.

The acoustic properties of the elastic foam samples were investigated by combination of this multi-scale approach and engineering measurements, Fig. 3 (and Table 1 and Table 3). Using measured densities of $\mathrm{H}_{1}$ and $\mathrm{H}_{2}$, the sound absorbing behavior of the low-density foam was estimated using a limp model ${ }^{32}$, in a good agreement with the measurements in the impedance tube. The additional sound absorption generated using a limp model for the low density foam was determined to be due to an added rigid body motion of the overall sample. The sound transmission loss that dropped in the low frequency range with the resonance frequency were more elastically sensitive compared with sound absorbing properties whose essential behavior can be captured with a limp model.
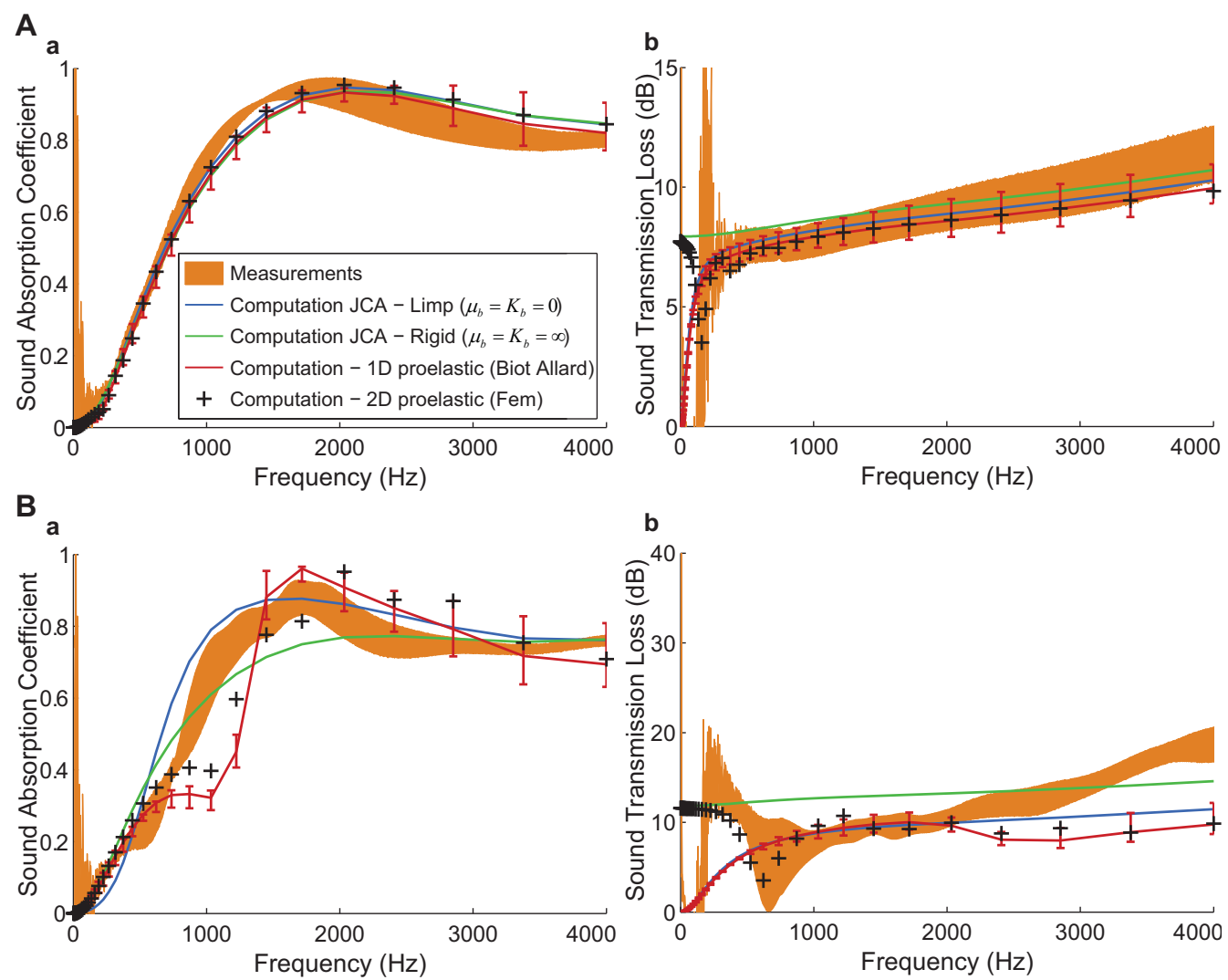

FIGURE 3. Acoustical properties of two different poroelastic foam samples, $H_{1}(A)$ and $H_{2}(B)$. 


\section{CONCLUSION}

Let us have an overall view of the results that were derived from this multi-scale approach with the poroelastic foam samples used in this paper (Fig. 1). For the sake of clarity, let us recall the procedure. The porosity $\phi$, permeability $k_{0}$ and ligament length $L$ are assumed to be known from measurements. In the multi-scale approach, the extension of the solid film was implemented at growing rates, while the unit cell was rescaled to account for the film frictional forces, until ligament length $L$ is converged. Macroscopic parameters are then computed from numerical homogenization and compared to the values that were measured at macro-scale and gathered in Table 1 and 3 . These later values serve in a sense as bridges between microstructure and acoustical macro-behavior with microphysical and micromechanical foundations. The numerical simulations are generally in good agreement with the standing wave tube measured values. The comparison for elastic properties is less impressive here since accurate values for the microscopic Young's modulus and Poisson ratio of the matrix were not yet available in the timeline of this study, while the estimation of the membrane thickness is rather rough at this stage. So, an important further step will be to refine the observation of membrane thickness from suitable observation means and to give a precise estimation of polyurethane Young's modulus by using means such as Mercury Intrusion Porosimetry (MIP) or nanoindentation techniques. Results for the sound absorption and transmission look much better when the circumferential constraint effect of the tube is taken into account through an axisymmetric FE code (Fig. 3). Therefore, the proposed multi-scale and multi-physics modeling approach of the acoustics of poroelastic foams provides a suitable strategy to characterize and optimize this class of materials. Note that this method provides new and complementary information when compared to inverse estimates of anisotropic open-cell foams. ${ }^{33}$ Considering the utility of cellular solids and foam structures in various applications, the versatility of periodic unit cell identification, and the chemical tunability of foam microstructures and mechanical functionality, this strategy can find ever broader application (biomedical and biomechanics, food industry, catalysis, etc.) because of added advantages of simple microstructure generation and parameterization, macroscopic properties estimation, and processability.

\section{REFERENCES}

1. J. F. Allard and N. Atalla, "Propagation of sound in porous media: Modelling sound absorbing materials," 2nd Ed. (Wiley, Chichester, 2009)

2. M. A. Biot, "Theory of propagation of elastic waves in a fluid-saturated porous solid. I. Low frequency range, ” J. Acoust. Soc. Am. 28, 168 (1956); M. A. Biot, "Theory of propagation of elastic waves in a fluid-saturated porous solid. II. Higher frequency range, ” J. Acoust. Soc. Am. 28, 179 (1956).

3. D. L. Johnson, J. Koplik, and R. Dashen, "Theory of dynamic permeability and tortuosity in fluid-saturated porous media," J. Fluid Mech. 176, 379 (1987).

4. Y. Champoux and J. F. Allard, "Dynamic tortuosity and bulk modulus in air-saturated porous media," J. Appl. Phys. 70, 1975 (1991).

5. P. Sheng and M. Y. Zhou, "Dynamic permeability in porous media," Phys. Rev. Lett. 61, 1591 (1988)

6. J. L. Auriault, "Dynamic behavior of a porous medium saturated by a Newtonian fluid, ” Int. J. Eng. Sci. 18, 775 (1980)

7. R. Burridge and J. B. Keller, "Poroelasticity equations derived from microstructure," J. Acoust. Soc. Am. 70, 1140 (1981)

8. I. Malinouskaya, V. V. Mourzenko, J.-F. Thovert, and P. M. Adler, "Wave propagation through saturated porous media, " Phys. Rev. E 77, 066302 (2008).

9. C. Boutin and C. Geindreau, "Periodic homogenization and consistent estimates of transport parameters through sphere and polyhedron packings in the whole porosity range," Phys. Rev. E 82, 036313 (2010).

10. L. J. Gibson and M. F. Ashby, "Cellular solids: Structure and Properties" (Cambridge University Press, Cambridge, 1988).

11. D. Weaire and S. Hutzler, "The Physics of Foams » (Oxford University Press, Oxford, 1999).

12. L. Gong, S. Kyriakides, W.-Y. Jang, "Compressive response of open-cell foams. Part I: Morphology and elastic properties," Int. J. Solids Struct. 42, 1355 (2005).

13. R. M. Sullivan, L. J. Ghosn, B. A. Lerch, “A general tetrakaidecahedron model for open-celled foams,” Int. J. Solids Struct. 45, 1754 (2008); R. M. Sullivan, L. J. Ghosn, B. A. Lerch, E. H. Baker, "Elongated Tetrakaidecahedron micromechanics model for space shuttle external tank foams," NASA/TP, 215137 (2009).

14. W.-Y. Jang, S. Kyriakides, A. M. Kraynik, "On the compressive strength of open-cell metal foams with Kelvin and random cell structures, ” Int. J. Solids Struct. 47, 2872 (2010).

15. P. Thiyagasundaram, B. V. Sankar, N. K. Arakere, "Elastic properties of open-cell foams with tetrakaidecahedral cells using finite element analysis," AIAA Journal 48, 818 (2010).

16. L. Boeckx, M. Brennan, K. Verniers, and J. Vandenbroeck, "A numerical scheme for investigating the influence of the three dimensional geometrical features of porous polymeric foam on its sound absorbing behavior," Acta Acust. Acust. 96, 239 (2010).

17. O. Doutres, N. Atalla, K. Dong, "Effect of the microstructure closed pore content on the acoustic behavior of polyurethane foams," J. Appl. Phys. 110, 064901 (2011). 
18. "Homogenization in mechanics of materials", Edited by M. Bornert, T. Bretheau, P. Gilormini (Wiley-Iste, 2008).

19. C. Perrot, F. Chevillotte, M. T. Hoang, G. Bonnet, F.-X. Bécot, L. Gautron, and A. Duval, "Microstructure, transport, and acoustic properties of open-cell foam samples: Experiments and three-dimensional numerical simulations," J. Appl. Phys. 111, 014911 (2012).

20. M. T. Hoang and C. Perrot, "Solid films and transports in cellular foams," J. Appl. Phys. 112, 054911 (2012).

21. M. T. Hoang and C. Perrot, "Identifying local characteristic lengths governing sound wave properties in solid foams," Submitted to J. Appl. Phys. (2012).

22. L. L. Beranek, “Acoustic impedance of porous materials," J. Acoust. Soc. Am. 13, 248 (1942).

23. M. R. Stinson and G. A. Daigle, "Electronic system for the measurement of flow resistance," J. Acoust. Soc. Am. 83, 2422 (1988).

24. J. L. Auriault, "Heterogeneous medium. Is an equivalent macroscopic description possible?,” Int. J. Eng. Sci. 29, 785 (1991).

25. Ph. Leclaire, L. Kelders, W. Lauriks, M. Melon, N. Brown, and B. Castagnède, "Determination of the viscous and thermal characteristic lengths of plastic foams by ultrasonic measurements in helium and air," J. Appl. Phys. 80, 2009 (1996).

26. Y. Atalla and R. Panneton, "Inverse acoustical characterization of open cell porous media using impedance tube measurements," Can Acoust. 33, 11 (2005).

27. E. Mariez, S. Sahraoui, and J. F. Allard, "Elastic constants of polyurethane foam's skeleton for Biot model," Proceedings of Internoise 96, pp. 951-954 (1996).

28. C. Langlois, R. Panneton and N. Atalla, "Polynomial relations for quasi-static mechanical characterization of isotropic poroelastic materials," J. Acoust. Soc. Am. 110, 3032 (2001).

29. L.D. Artavia and C.W. Macosko, "Polyurethane flexible foam formation," (pp. 22-55); R.D. Priester and R. B. Turner, "The morphology of flexible polyurethane matrix polymers," (pp. 78-103); in Low density cellular plastics: physical basis of behavior, Edited by N.C. Hilyard and A. Cunningham (Chapman \& Hall, London, 1994).

30. J. Lecomte-Beckers, "Cours de Physique des Matériaux ", Université de Liège (2009).

31. D. Pilon, R. Panneton, and F. Sgard, "Behavioral criterion quantifying the edge-constrained effects on foams in the standing wave tube », J. Acoust. Soc. Am. 114, 1980 (2003).

32. R. Panneton, "Comments on the limp frame equivalent fluid model for porous media," J. Acoust. Soc. Am. 122, EL217 (2008).

33. N.-E. Hörlin and P. Göransson, Weak, "anisotropic symmetric formulations of Biot's equations for vibro-acoustic modelling of porous elastic materials,” Int. J. Numer. Meth. Eng. 84, 1519 (2010); P. Göransson, R. Gustavino, and N.-E. Hörlin, “ Measurement and inverse estimation of 3D anisotropic flow resistivity for porous materials », J. Sound and Vibration 327, 354 (2009); J. Cuenca and P. Göransson, "Inverse ertimation of the elastic and anelastic properties of the porous frame of anisotropic open-cell foams," J. Acoust. Soc. Am. 132, 621 (2012). 